\title{
Soft Computing Applications in Actuarial Science
}

\author{
Arnold F. Shapiro \\ (afs1@psu.edu)
}

Smeal College of Business, Penn State University, University Park, PA 16802, USA

\begin{abstract}
The purpose of this article is to provide an overview of soft computing applications in actuarial science. Soft computing (SC) refers to modes of computing in which precision is traded for tractability, robustness and ease of implementation. For the most part, SC encompasses the technologies of fuzzy logic, genetic algorithms, and neural networks, and it has emerged as an effective tool for dealing with control, modeling, and decision problems in complex systems. The paper ends with a general comment on the study.
\end{abstract}

\section{Acknowledgments}

This work was supported in part by the Robert G. Schwartz Faculty Fellowship at the Penn State University and a grant of the Committee on Knowledge Extension and Research (CKER) of the Society of Actuaries. 


\section{Soft Computing Applications in Actuarial Science}

\section{Introduction}

Soft computing (SC) is a concept that was introduced by Zadeh (1992), the discoverer of fuzzy logic. He envisioned SC as being ...concerned with modes of computing in which precision is traded for tractability, robustness and ease of implementation. For the most part, SC encompasses the technologies of fuzzy logic, genetic algorithms, and neural networks, and it has emerged as an effective tool for dealing with control, modeling, and decision problems in complex systems. Briefly, fuzzy logic is used to deal with imprecision and uncertainty, genetic algorithms are used for search and optimization, and neural networks are used for learning and curve fitting. In spite of these dichotomies, there are natural synergies between these technologies.

The purpose of this article is to provide an overview of soft computing applications in actuarial science. The review is not meant to be exhaustive, rather it is intended to show where soft computing has made an inroad. The paper ends with a general comment on the study.

\section{Soft Computing Applications}

This section provides an overview of representative articles on soft computing applications in actuarial science. First, neural network applications are discussed, then the discussion turns to fuzzy logic applications, and finally the focus turn to applications involving genetic algorithms. This dichotomy is not absolute, of course, since hybrid systems are discussed throughout the section.

\section{Neural Networks Applications}

A survey of neural network applications in actuarial science is provided in this segment of the pager. For the most part, these areas of application include classification, asset and investment models, insolvency studies, and mortality and morbidity studies. Since this is a relatively new area of analysis, a number of the studies also include comparisons with rival approaches.

\section{Classification}

Classification is fundamental to the insurance business. On the one hand, risks need to be properly classified and segregated for pricing purposes, while on the other hand, classification is basic to the underwriting of potential coverages. This section discusses three representative classification areas where NN have been employed: construction bond underwriting, the underwriting of life insurance applicants, and the detection of fraudulent insurance claims. 
Bakheet (1995) used the backpropagation form of the feed forward NN as the pattern classification tool in construction bond underwriting. Surety companies bond construction contractors based on the evaluation of a set of decision factors, such as character, capital, capacity, and continuity. The study used an independent pattern classification module for these four factors and an integrated pattern classification model. The conclusion was that the model was an efficient method for handling the evaluation.

Vaughn et. al. (1997) used a multilayer perceptron network to classify applicants for whole life insurance into standard and non-standard risk. They then used a knowledge discovery method to identify the significant, or key, inputs that the network uses to classify applicants. The ranking of these inputs enables the knowledge learned by the network during training to be presented in the form of data relationships and induced rules which show that the network learns sensibly and effectively when compared with the training data set.

Brockett et. al. (1998) used a kohonen s self-organizing feature map (SOFM) to uncover automobile bodily injury claims fraud in the insurance industry and a feed forward neural networks using a back propagation algorithm to validate the feature map approach. The focus of the study was to determine whether a claim is fraudulent and the level of suspicion of fraud associated with the claim record file. Their conclusion was that the consistency and reliability of the fraud assessment of the SOFM exceeds that of both an insurance adjuster and an insurance investigator in assessing the suspicion level of bodily injury (BI) claims, and they suggested that a similar methodology can be used for detecting fraud in Medicare claims.

\section{Asset and Investment Models}

The analysis of assets and investments is a major component in the management of an insurance enterprise. Of course, this is true of any financial intermediary, and many of the functions performed are uniform across financial companies. Thus, insurers are involved with market and individual stock price forecasting, the forecasting of currency futures, credit decision-making, forecasting direction and magnitude of changes in stock indexes, and so on. This section provides an overview of some of the studies performed in these areas, although not necessarily in an insurance context. Numerous other examples are contained in Refenes (1995).

Boero and Cavalli (1996) investigate a NN model for forecasting the exchange rate between the Spanish peseta and the US dollar. Their goal was to examine whether potentially highly nonlinear neural network models outperform traditional methods or give at least competitive results. To this end, they compare the performance of $\mathrm{NN}$ with four linear models, a random walk process and three different specifications based on the purchasing power parity theory. They found mixed results. In experiments with quarterly data, they found no advantage in the use of NNs for forecasting the exchange rate, while the performance of the NNs clearly improves when they are trained on monthly data. 


\section{Insolvency}

Because of the inherent nature of an insurance contract, the insurance industry is regulated, and one of the primary considerations of regulators is insurer solvency. In the past, most studies of insurance company failure prediction used statistical methods such as multiple discriminant analysis (MDA) and logistic regression analysis, ID3, and CART. Recently, NNs were brought to bear on the problem of insurance company insolvency. This section presents an overview of four such studies and their conclusions with respect to traditional statistical methods. The first two papers address insolvency from the perspective of a nonlife insurer while the last two articles focus on life insurers.

One of the earlier studies was conducted by Park (1993), who used a neural network designed by a genetic algorithm to predict the bankruptcy of insurance companies, and to compare the results with those obtained using discriminant analysis, logistic regression, ID3, and CART. Using a genetic algorithm to suggests the optimal structure and network parameters was found to be superior to using randomization in the design of the neural network, a finding that was confirmed in a study of bank insolvencies. The major conclusion of the study was that the robust neural network model outperformed the statistical models. The study also addressed the issue of conflicts in model performance. While previous studies attribute the performance of a model to the application domain, this study argues that the performance of a model is affected by the distribution of independent variables and that the performance of the models can be compared from the view of task characteristics.

A similar study was undertaken by Brockett et. al. (1994), who used a three-layer feed-forward, back-propagation, neural network to develop an early warning system for U.S. property-liability insurers two years prior to insolvency. The results of the neural network method were compared with those of discriminant analysis, the rating of an insurer rating organization, and the ratings of an national insurance regulatory body. The conclusion was that the neural network approach outperformed the discriminant analysis and did far better than the rating organization, and the regulators. Generally speaking, the neural network results show high predictability and generalizability, suggesting the usefulness of this method for predicting future insurer insolvency.

Huang et. al. (1995) use an NN optimized with a genetic algorithm to forecast financial distress in life insurers, and compare the forecasts to those obtained using discriminant analysis, k-nearest neighbor, and logit analysis. The data is limited to Insurance Regulatory Information System Ratios for the total population of insurers. They conclude that the NN dominates the other two methods for both in-sample fit and out of sample forecasting.

Jang (1997) undertook a similar comparative analysis. Here the focus was on a comparison of multiple discriminant analysis (MDA) and logistic regression analysis with NN based on back-propagation (BP), learning vector quantization (LVQ) and self-organizing feature maps (SOM). As with the Huang et. al. (1995) study, the back-propagation (BP) and learning vector quantization (LVQ) outperform the traditional statistical approaches for all data sets with a 
consistent superiority across the different evaluation criteria. The self-organizing feature map (SOM) supported these findings by showing the distinct areas of bankrupt and non-bankrupt companies geographically, which suggests that the easier visual interpretation SOM can be used as a management tool by both insurance regulators and the companies themselves.

\section{Mortality and Morbidity Studies}

The basis of the life and health insurance premiums are the mortality and morbidity rates, so it is not surprising that we are beginning to see NNs applied to the determination of these rates. Three examples of this type of application are related to intensive care, dental caries, and inhospital complications.

Tu (1993) compared a neural network and logistic regression models for predicting length of stay in the intensive care unit (ICU) following cardiac surgery. This is important from an actuarial perspective because improving the efficiency of hospitals has a direct impact on insurance costs. Structurally, the training set and test set consisted of approximately 700 patients each, and five unique risk strata were created using each model. The advantages and disadvantages of each modeling technique was explored, with the conclusion was that either model could potentially be used as a risk stratification tool for counseling patients and scheduling operations.

Saemundsson (1996) used NN to addressed the problem of identifying individuals at risk of developing dental caries, an issue which has implications for dental care insurance. The focus of the research was to compare the predictive capabilities of clinicians, NN and logistic regression. The study revealed that human prediction of dental disease was at a comparable level of performance to logistic regression, and that the best predictive performance of any method in the study was reached by eliminating some of the uncertainty introduced by dental treatment interventions into the natural process of dental caries and by using an ANN prediction model with human prediction as one of the inputs.

An example of the use of NN for a mortality study was provided by Ismael (1999), who investigated the feasibility of using neural network technology to reliably predict in-hospital complications and 30-day mortality for acute myocardial infarction (MI) patients. A database of 20,873 American patients who suffered an acute MI between 1990 and 1993 was used in this analysis. Twenty-two history and physical variables were analyzed as predictors for 16 distinct complications. The specific steps included a feasibility stage, during which an extensive univariate and bivariate analysis was performed on the database to determine the feasibility of the study, a training stage, during which 16 separate feedforward, backpropagation neural networks were trained and analyzed, one for each complication, and a testing stage that calculated a score for the patients representing their likelihood of having any complication. The index of discrimination for each of the neural networks, as well as for the scoring system, was defined to be the area underneath their respective ROC curves. The conclusion of the study was that the neural networks proved to be successful in predicting only four of the 16 specific 
complications: death within 30 days, shock, asystole, and congestive heart failure or pulmonary edema. The scoring system was found to have had a rather low discriminatory ability

\section{Fuzzy Logic Applications}

This section reports on four major insurance areas where fuzzy logic has been implemented: pricing, insurance company assets, projected liabilities, and underwriting. Readers who are also interested in potential areas of applications will find a large number of them mentioned in Ostaszewski (1993). Following Zadeh (1994: 192), in this article the term fuzzy logic (FL) is used in the broad sense where it is essentially synonymous with fuzzy set theory.

\section{Pricing}

Young (1996b) described how FL can be used to make pricing decisions in group health insurance that consistently consider supplementary data, including vague or linguistic objectives of the insurer, which are ancillary to statistical experience data. Using group health insurance data from an insurance company, an illustrative competitive rate changing model is built, that employs fuzzy constraints exclusively to adjust insurance rates. Young did not necessarily advocate using such a model without considering experience studies but presented the simplified model to demonstrate more clearly how to represent linguistic rules.

The foregoing analysis was extended to include claim experience data in Young (1997). In this case, the author describes step-by-step how an actuary/decision maker can use FL to adjust workers compensation insurance rates. The supplementary data may be financial or marketing data or statements that reflect the philosophy of the actuary's company or client.

The final example of this section is a study by Cummins and Derrig (1997), who used FL to address the financial pricing of property-liability insurance contracts. Both probabilistic and nonprobabilistic types of uncertainty in the pricing and underwriting accept/reject context are incorporated. The authors focus primarily on FL aspects needed to solve the insurance-pricing problem, and in the process "fuzzify" a well-known insurance financial pricing model, provide numerical examples of fuzzy pricing, and propose fuzzy rules for project decision-making. They conclude that FL can lead to significantly different pricing decisions than the conventional approach.

\section{Underwriting}

The first recognition of FL applicability to the problem of individual insurance underwriting was due to DeWit (1982). Although the analysis was not detailed, DeWit realized that an insurance contract is based on its (risk) premium estimated as exactly as possible, and also on a more intuitive kind of experience which is expressed by the company's underwriting practice.

Lemaire (1990) extended DeWit (1982) to a more extensive agenda for FL in insurance theory, most notably in the financial aspects of the business. He discussed three problems: the definition 
of a preferred policyholder in life insurance, the selection of an optimal excess of loss retention in a reinsurance application, and the computation of the fuzzy premium for a pure endowment policy.

Young (1993 ) used fuzzy sets to model the selection process in group health insurance. Applying the model of fuzzy multi-stage decision processes of Bellman and Zadeh (1970) to group health underwriting, she defines fuzzy sets that characterize groups that are good underwriting risks. First single-plan underwriting is considered and then the study is extended to multiple-option plans.

Derrig and Ostaszewski (1995) extended the previous work by showing how fuzzy set theory can be combined with cluster analysis and applied to risk and claim classification. This fuzzy clustering that provides an alternative way to view risk classification. Their focus was on applying fuzzy clustering algorithms to problems of auto rating territories and fraud detection and on the classification of insurance claims according to their suspected level of fraud. Conceptually, when the fuzzy cluster algorithm is applied to classification of rating territories, the clusters are the risk classes, and the degree of belief that each territory belongs to a given cluster (risk class) is quantified as a real-valued number between zero and one.

Jablonowski (1997) examines the problems for risk managers associated with knowledge imperfections and describes how FL can be used to deal with situations when there is a lack of knowledge. Not much detail was provided, however.

A example of a different underwriting dimension was provided by Kieselbach (1997) who used FL for systematic failure analysis. The role of the research was to analyze structure failures in order to prevent similar cases in the future, clarify responsibilities, add to experience, contribute to design theory. The possible advantages of using fault tree analysis as a tool for systematic failure analysis was also discussed. The failure of the railing of a scaffold, which led to an accident, was discussed as an example of the feasibility of this kind of systematic approach and the application of FL to failure analysis

Most reported studies of applications of FL in insurance underwriting were written by academicians. Two exception are the studies of Erbach and Seah (1993) and Horgby et. al. (1997). One of the first practical insurance application of FL took place in Canada in 1987, and involved the development of Zeno, a prototype life automated underwriter using a mixture of fuzzy and other techniques. According to Erbach and Seah (1993), it was carried as far as developing an underwriter (coded in $\mathrm{C}++$ ) that ran on portable computers, and, with the aid of electronic links to the home office, it was intended to make turnaround on cases complicated enough to require human intervention a matter of minutes.

The other underwriting application was provided by Horgby et. al. (1997), who applied FL to medical underwriting of life insurance applicants. In a step-by-step fashion, the authors show how expert knowledge about underwriting diabetes mellitus in life insurance can be processed 
for a fuzzy inference system. The article was based on one of the first computer-based fuzzy underwriting system implemented in industry and, given the success of the application, the authors conclude that techniques of fuzzy underwriting will become standard tools for underwriters in the future.

\section{Asset and Investment Models}

As mentioned in the section on neural networks, a good deal of the research on allocation of assets and investment analysis can be drawn from research with respect to other financial intermediaries. Two interesting sources in this regard are Chorafas (1994, chapters 8-10), which describes several applications of FL in finance, and Siegel, de Korvin, and Omer (1995), which describe many applications of fuzzy set from an accounting perspective.

Two interesting applications which are unique to the insurance area involve immunization and insurance company taxation. The following is an overview of these studies.

Chang and Wang (1995) develop fuzzy mathematical analogues of the classical immunization theory and the matching of assets and liabilities. Essentially, they reformulate concepts about immunization and the matching of assets and liabilities into fuzzy mathematics. This approach offers the advantages of flexibility, improved conformity with situations encountered in practice and the extension of solutions.

Derrig and Ostaszewski (1997) view the insurance liabilities, properly priced, as a management tool of the short position in the government tax option. In this context, they illustrate how FL can be used to estimate the effective tax rate and after-tax rate of return on a property-liability insurance company s asset and liability portfolio. To accomplish this, they modeled critical parameters of underwriting and investment as fuzzy numbers, which lead to a model of uncertainty in the tax rate, rate of return, and the asset-liability mix.

\section{Projected Liabilities}

The evaluation of projected liabilities is a fundamental function of an insurer. As was mentioned in the section on neural networks, mortality and morbidity studies are crucial to the life and health insurance industry. In the property and casualty industry the issue is property damage and liability. This section presents an overview of three diverse studies in these areas.

Boissonnade (1984) uses pattern recognition and FL in the evaluation of seismic intensity and damage forecasting, and for the development of models to estimate earthquake insurance premium rates and insurance strategies. The influences on the performance of structures include quantifiable factors, which can be captured by probability models, and nonquantifiable factors, such as construction quality and architectural details, which are best formulated using fuzzy set models. Accordingly, two methods of identifying earthquake intensity are presented and compared. The first method is based on the theory of pattern recognition where a discriminative function is developed based on Bayes' criterion and the second method applies the FL. 
Cummins and Derrig (1993) study fuzzy trends in property-liability insurance claim costs. The essence of the study is that they emphasize the selection of a "good" forecast where goodness is defined using multiple criteria that may be vague or fuzzy rather than a forecasting model. FL is proposed as a mechanism for combining forecasts from alternative models using multiple fuzzy criteria. The methodology they propose to calculate a trend factor in property-liability insurance was first suggested by Bellman and Zadeh (1970), and they calculate several possible trends using accepted statistical procedures. For each trend, they determine the degree to which the estimate is good by intersecting several fuzzy goals. They suggest that one may choose the trend that has the highest degree of goodness and propose that a trend that accounts for all the trends can be calculated by forming a weighted average using the membership degrees as weights. They conclude that FL provides an effective method for combining statistical and judgmental criteria in actuarial decision making.

A final perspective is provided by Zhao (1996), who addressed the issues of maritime collision prevention and liability. The essence of the analytical portion of the study was the use of fuzzy programming methods to build decision making simulation models and the development of an automatic collision avoidance decision making system using FL and neural networks.

\section{Genetic Algorithm Applications}

Genetic algorithms have become a popular alternative to linear programming and other optimization routines because of their power and ease of use. Moreover, as mentioned previously, an important use of GA is their role in SC hybrid systems. Insurance applications have included classification, optimizing the competitiveness of an insurance product, and asset allocation. This section provides a brief overview of examples of these studies. The discussion is restricted to a sample of papers where GAs is the only soft computing technique employed.

\section{Classification}

Lee and Kim (1999) use GAs to refine the classification system of Korean private passenger automobile insurance. The study was based on a sample of 20,000 cases randomly selected from automobile policies in force at the largest auto insurer in Korea, which is divided into two sets for training and testing purposes. The research employed a hybrid learning methodology that integrates genetic algorithms and decision tree learning. First, the GA was used to explore the space of all possible subsets of a large set of candidate discriminatory variables; then the candidate variables subsets were evaluated using a decision tree induction algorithm to produce a classifier from the given variables and the training data. As a benchmark, the classification performance of this technique was compared with that obtained using a logit model.

\section{Asset Allocation}

Wendt (1995) used a GA to built a portfolio efficient frontier (a set of portfolios with optimal combinations of risk and returns). The underlying data consisted of 250 scenarios of annual 
returns for eight asset classes. To evaluate the GA process, the final GA output is compared to the efficient frontier created by a sophisticated nonlinear optimizer. After about 50 cycles, the GA finds portfolios very close to the efficient frontier generated by the nonlinear optimizer.

\section{Competitiveness of the Insurance Products}

Tan (1997) begins the analysis with a Monte Carlo simulation, which is used to develop a flexible framework to measure the profitability, risk, and competitiveness of insurance products. A genetic algorithm is then used to seek the optimum asset allocations that form the profitabilityrisk-competitiveness frontier and to examine the profitability, risk, and competitiveness tradeoffs. The paper shows how to select the appropriate asset allocation and crediting strategy in order to position the product at the desired location on the profitability-risk-competitiveness spectrum.

\section{Comment}

The purpose of this paper has been to provide the reader with an overview of where SC has been implemented in the actuarial applications. While it is clear that SC has made inroads into many facets of the business, it also seems clear that there are enormous opportunities for additional applications. SC is a collection of methodologies whose focus is the exploitation of the tolerance for imprecision, uncertainty, robustness and low solution cost, characteristics which describe many of the outstanding problem areas of the business. Hence, almost surely, SC will be an important tool for actuarial analysis in the new millennium.

\section{References}

Anders, U. 1996. "Statistical Model Building for Neural Networks," 6th AFIR Colloquium.

Bakheet, M. T. 1995. "Contractors' Risk Assessment System (Surety, Insurance, Bonds, Construction, Underwriting) Ph.D. Dissertation, Georgia Institute of Technology.

Barber, J. C. 1995. "Genetic Algorithms as Tools for Optimization," Risks and Rewards, December.

Barber, J. C. 1996. "Neural Nets in Finance: An Overview," Risks and Rewards, March.

Bellman, R. and L. A. Zadeh. 1970. "Decision-Making in a Fuzzy Environment," Management Science, Vol. 17, pp.141-164.

Bishop, C. M. 1995. Neural Networks for Pattern Recognition. Clarendon Press.

Boero, G. and E. Cavalli. 1996. "Forecasting the Exchange Rage: A Comparison Between 
Econometric and Neural Network Models," AFIR, Vol. II, 981.

Boissonnade, A. C. 1984. "Earthquake Damage and Insurance Risk." Ph.D. Dissertation, Stanford University.

Brockett, P. L., W. W. Cooper, L. L. Golden and U. Pitaktong. 1994. "A Neural Network Method for Obtaining an Early Warning of Insurer Insolvency," Journal of Risk and Insurance, Vol. 61, No. 3, 402.

Brockett, P. L., Xiaohua Xia and Richard A. Derrig. 1998. "Using Kohonen's Self-Organizing Feature Map to Uncover Automobile Bodily Injury Claims Fraud," Journal of Risk and Insurance, Vol. 65, No. 2, 245.

Chang, C. and P. Wang. 1995. "The Matching of Assets and Liabilities with Fuzzy Mathematics," 25th International Congress of Actuaries, 123.

Chorafas, D. N. 1994. Chaos Theory In The Financial Markets: Applying Fractals, Fuzzy Logic, Genetic Algorithms. Chicago: Probus Publishing Company.

Cummins, J. D. and R. A. Derrig. 1993. "Fuzzy Trends in Property-Liability Insurance Claim Costs," Journal of Risk and Insurance, Vol. 60, pp. 429-465.

Cummins, J. D. and R. A. Derrig. 1997. "Fuzzy Financial Pricing of Property-Liability Insurance," North American Actuarial Journal, Vol 1, No. 4, 21.

Deboeck, G., Editor. 1994. Trading on the Edge: Neural, Genetic, and Fuzzy Systems for Chaotic Financial Markets. New York: John Wiley and Sons.

Derrig, R. A. and K. Ostaszewski. 1995. "Fuzzy Techniques of Pattern Recognition in Risk and Claim Classification," Journal of Risk and Insurance, Vol. 62, pp. 447-482.

Derrig, R. A. and K. Ostaszewski. 1997. "Managing the Tax Liability of a Property-Liability Insurance Company," Journal of Risk and Insurance, Vol. 64, 694.

DeWit, G. W. 1982. "Underwriting and Uncertainty," Insurance: Mathematics and Economics, Vol. 1, pp. 277-285.

Driankov, D., H. Hellendoom and M. Reinfrank. 1993. An Introduction to Fuzzy Control. Berlin: Springer-Verlag.

Dubois, D. and H. Prade. 1980. Fuzzy Sets and Systems: Theory and Applications. San Diego, CA: Academic Press.

Dubois, D. and H. Prade. 1988. Possibility Theory: An Approach to the Computerized 
Processing of Uncertainty. New York: Plenum Press.

Dunis, C., Editor. 1996. Forecasting Financial Markets: Exchange Rates, Interest Rates and Asset Management. New York: John Wiley \& Sons.

Eberhardt, R. C. and R. W. Dobbins. 1990. Neural Network PC Tools: A Practical Guide. New York: Academic Press.

Erbach, D. W. and E. Seah. 1993. Discussion of "The Application of Fuzzy Sets to Group Health Underwriting" by V. R. Young, Transactions of the Society of Actuaries, 45, 585.

Goldberg, D. E. 1989. Genetic Algorithms in Search, Optimization, and Machine Learning. Addison-Wesley.

Holland, J. H. 1975. Adaptation in Natural and Artificial Systems. Univ. Michigan Press, Ann Arbor.

Horgby, P., R. Lohse and N. Sittaro. 1997. "Fuzzy Underwriting: An Application of Fuzzy Logic to Medical Underwriting," Journal of Actuarial Practice, Vol. 5, No. 1, 79.

Huang, C., R. E. Dorsey and M. A. Boose. 1995. "Life Insurer Financial Distress Prediction: A Neural Network Model," Journal of Insurance Regulation, Winter, Vol. 13, No 2, pp. 131-167.

Ismael, M. B. 1999. "Prediction of Mortality and In-hospital Complications for Acute Myocardial Infarction Patients Using Artificial Neural Networks." Ph.D. Dissertation, Duke University.

Jablonowski, M. 1997. "Modeling Imperfect Knowledge in Risk Management and Insurance," Risk Management and Insurance Review, Vol. 1, No. 1, 98.

Jang, J. 1997. "Comparative Analysis of Statistical Methods and Neural Networks for Predicting Life Insurers' Insolvency (Bankruptcy)" Ph.D. Dissertation, University of Texas at Austin.

Kandel, A. and G. Langholz. 1994. Fuzzy Control Systems. Boca Raton, FL: CRC Press.

Kaufmann, A. and M. Gupta. 1985. Introduction to Fuzzy Arithmetic: Theory and Applications. New York: Van Nostrand-Reinhold.

Kieselbach, R. 1997. "Systematic Failure Analysis Using Fault Tree and Fuzzy Logic," Technology, Law and Insurance, No. 2, 13-20.

Klir, G. J. and T. A. Folger. 1988. Fuzzy Sets, Uncertainty, and Information. Englewood Cliffs, NJ: Prentice Hall. 
Kohonen, T. 1982. "Self-Organizing Formation of Topologically Correct Feature Maps." Biological Cybernetics,43: 59-69.

Kohonen, T. 1988. "Self-Organizing Feature Maps. Self-Organizing and Associative Memory, 2nd ed. Berlin, Heidelberg, Germany: Spring-Verlag.

Kohonen, T. 1990. "The Self-Organizing Map," Proceedings of the IEEE 78(9): 1464-1480.

Lee, B. and M. Kim. 1999, Application of Genetic Algorithm to Automobile Insurance for Selection of Classification Variables: The Case of Korea. Paper presented at the 1999 Annual Meeting of the American Risk and Insurance Association.

Lemaire, J. 1990. "Fuzzy Insurance," ASTIN Bulletin, Vol. 20, No. 1, pp. 33-55.

Ostaszewski, K. 1993. Fuzzy Set Methods in Actuarial Science. Schaumburg, IL: Society of Actuaries.

Park, J. 1993. Bankruptcy Prediction of Banks and Insurance Companies: an Approach Using Inductive Methods. Ph.D. Dissertation, University of Texas at Austin.

Refenes, A-P. 1995. Neural Networks in the Capital Markets. New York: John Wiley \& Sons.

Saemundsson, S. R. 1996. "Dental Caries Prediction by Clinicians and Neural Networks." Ph.D. Dissertation, University of North Carolina at Chapel Hill.

Siegel, P. H., A. de Korvin and K. Omer, eds. 1995. Applications of Fuzzy Sets and the Theory of Evidence to Accounting. Greenwich, Conn.: JAI Press.

Shapiro, A. F. (2000) A Hitchhiker s Guide to the Techniques of Adaptive Nonlinear Models, Insurance: Mathematics and Economics 26, (2000), 119-132.

Shapiro, A. F. (2000) Implementing Adaptive Nonlinear Models, Insurance: Mathematics and Economics 26, (2000), 289-307.

Shepherd, A. J. 1997. Second-Order Method for Neural Networks. Springer.

Smith, M. 1993. Neural Networks for Statistical Modeling. Van Nostrand Reinhold.

Tan. 1997. "Seeking the Profitability-Risk-Competitiveness Frontier Using a Genetic Algorithm," Journal of Actuarial Practice, 5, No. 1, 49.

Topping, B. H. V. and A. Bahreininejad. 1997. Neural Computing for Structural Mechanics. Edinburgh: Saxe-Coburg Publications. 
Tu, J. V. 1993. A Comparison of Neural Network and Logistic Regression Models for Predicting Length of Stay in the Intensive Care Unit Following Cardiac Surgery." Ph.D. Dissertation, University of Toronto.

Vaughn, M. L., E. Ong and S. J. Cavill. 1997. "Interpretation and Knowledge Discovery from a Multilayer Perceptron Network that Performs Whole Life Assurance Risk Assessment," Neural Computing and Applications, 6:201-213.

Von Altrock, C. 1997. Fuzzy Logic and NeuroFuzzy Applications in Business and Finance. Prentice-Hall.

Vonk, E., L. C. Jain and R. P. Johnson. 1997. Automatic Generation of Neural Network Architecture Using Evolutionary Computation, Word Scientific.

Wendt, R. Q. 1995. "Build Your own GA Efficient Frontier," Risks and Rewards, December.

Young, V. R. 1993. "The Application of Fuzzy Sets to Group Health Underwriting," Transactions of the Society of Actuaries, Vol. 45, pp. 551-590.

Young, V. R. 1996a. "Fuzzy Subsethood," Fuzzy Sets and Systems, Vol. 77, pp. 371-384.

Young, V. R. 1996b. "Insurance Rate Changing: A Fuzzy Logic Approach," Journal of Risk and Insurance, Vol. 63, pp. 461-483.

Young, V. R. 1997. "Adjusting Indicated Insurance Rates: Fuzzy Rules that Consider Both Experience and Auxiliary Data," Proceedings of the Casualty Actuarial Society, 84, 734.

Zadeh, L. A. 1965. "Fuzzy Sets," Information and Control, Vol. 8, pp.338-353.

Zadeh, L. A. 1975, 1976. "The Concept of Linguistic Variable and its Application to Approximate Reasoning (Parts 1-3)," Information Sciences, Vol. 8, pp. 199-249, 301-357, and Vol. 9, pp. 43-80.

Zadeh, L. A. 1992. Foreword of the Proceedings of the Second International Conference on Fuzzy Logic and Neural Networks, pp. Xiii-xiv, Iizuka, Japan.

Zadeh, L. A. 1994. The Role of Fuzzy Logic in Modeling, Identification and Control, Modeling Identification and Control, 15(3), 191.

Zhao, J. 1996. "Maritime Collision and Liability." Ph.D. Dissertation, University of Southampton.

Zimmermann, H. J. 1987. Fuzzy Sets, Decision Making, and Expert Systems. Boston, MA: Kluwer Academic Publishers. 